\title{
Resultants of Chebyshev Polynomials
}

\author{
Jemal Gishe and Mourad E. H. Ismail
}

\begin{abstract}
Recently, K. Dillcher and K. B. Stolarsky [Trans. Amer. Math. Soc. 357 (2004), 965 - 981] used algebraic methods to evaluate the resultant of two linear combinations of Chebyshev polynomials of the second kind. In this paper we give an alternative method of computing the same resultant and resultants of more general combinations of Chebyshev polynomials of the second kind. We also consider resultants of linear combinations of Chebyshev polynomials of the first kind.
\end{abstract}

Keywords. Chebyshev polynomials, resultants, discriminants, lowering (annihilation) operators

Mathematics Subject Classification (2000). Primary 12E05, secondary 33C45, $13 \mathrm{P} 05$

\section{Introduction}

A resultant is a scalar function of two polynomials which is non-zero if and only if the polynomials are relatively prime. The theory of resultants is an old and much studied topic in what used to be called the theory of equations [5]. Resultants are interesting for many reasons. They can be used in matrix theory and in problems on locations of roots of polynomials. This is relevant to applications in linear control systems, in robotics and computer aided geometric design. There are many results on the theoretical properties of resultants especially in relation to algebraic geometry. For history and details of some of the applications of resultants, see $[3,4,7,8]$. Discriminants are special resultants and are useful in Ring Theory. In electrostatic equilibrium problems (see [10]), the discriminant represents the interaction free energy at equilibrium of a Coulomb gas model. Two noteworthy results are Apostol's evaluation of the resultant of two cyclotomic polynomials [2] and Roberts' [12] recent evaluation of discriminants of certain polynomials which appear in Painlevé analysis.

J. Gishe: Department of Mathematics, Western Kentucky University, Bowling Green, KY 42101-1078; jemal.gishe@wku.edu

M. Ismail: Department of Mathematics, University of Central Florida, Orlando, FL 32816; ismail@math.ucf.edu 
In this paper we compute resultants of linear combinations of Chebyshev polynomials. The resultant is expressible in terms of Chebyshev polynomials whose coefficients and arguments are rational functions of the coefficients in the linear combinations. The resultant of two two- term linear combinations of Chebyshev polynomials of the second kind is given in the recent work of Dilcher and Stolarsky [6]. They used algebraic properties of resultants with respect to division, and Euclidean algorithms for polynomials. We used a simple method which enabled us to compute resultants of a more general combination of Chebyshev polynomials. In Section 2 we compute resultants of different combinations of Chebyshev polynomials of second kind and in Section 3 we prove analoguous results involving Chebyshev polynomials of the first kind.

We follow classical definition of resultants, namely if

$$
A(x)=a_{n} \prod_{k=1}^{n}\left(x-x_{k}\right) \quad \text { and } \quad B(x)=b_{m} \prod_{j=1}^{m}\left(x-y_{j}\right)
$$

then the resultant of $A$ and $B$ is $\operatorname{Res}(A, B)=a_{n}^{m} b_{m}^{n} \prod_{k=1}^{n} \prod_{j=1}^{m}\left(x_{k}-y_{j}\right)$. It follows that

$$
\operatorname{Res}(A, B)=a_{n}^{m} \prod_{j=1}^{n} B\left(x_{j}\right) .
$$

The following useful theorem is due to I. Schur, see [13, $\$ 6.71]$.

Theorem 1.1. Let $\left\{p_{n}(x)\right\}$ be a sequence of polynomials satisfying the recurrence formula

$$
p_{n}(x)=\left(a_{n} x+b_{n}\right) p_{n-1}(x)-c_{n} p_{n-2}(x), \quad n=2,3, \ldots,
$$

and the intial conditions $p_{0}(x)=1, p_{1}(x)=a_{1} x+b_{1}$. Assume that $a_{1} a_{n} c_{n} \neq 0$ for $n>1$ and let $\left\{x_{j, n}\right\}$ be the zeros of $p_{n}(x)$. Then,

$$
\Delta_{n}:=\prod_{j=1}^{n} p_{n-1}\left(x_{j, n}\right)=(-1)^{\frac{n(n-1)}{2}} \prod_{j=1}^{n}\left\{a_{j}^{n-2 j+1} c_{j}^{j-1}\right\}, \quad n=1,2,3, \ldots
$$

Our approach is as follows. Assume $\left\{r_{n}(x)\right\}$ and $\left\{s_{n}(x)\right\}$ are sequences of polynomials such that $r_{n}$ and $s_{n}$ have exact degree $n$ for all $n, n \geq 0$. Construct functions $A_{n}(x)$ and $B_{n}(x)$ such that $s_{n-1}(x)=A_{n}(x) r_{n-1}(x)+B_{n}(x) r_{n}(x)$. With $r_{n}(x)=\rho_{n} \prod_{j=1}^{n}\left(x-\zeta_{j, n}\right)$ then

$$
\operatorname{Res}\left\{r_{n}(x), s_{n-1}(x)\right\}=\rho_{n}^{n-1}\left[\prod_{j=1}^{n} r_{n-1}\left(\zeta_{j, n}\right)\right]\left[\prod_{j=1}^{n} A_{n}\left(\zeta_{j, n}\right)\right] .
$$

When $r_{n}(x)$ satisfies a three term recurrence relation of the form (1.2) then Theorem 1.1 evaluates the first product on the last line of (1.4). The product $\prod_{j=1}^{n} A_{n}\left(\zeta_{j, n}\right)$ is evaluated on a case-by-case basis. 
The motivation for our approach came from the work [9] on discriminants of general orthogonal polynomials. In Section 2 we rederive the Dilcher-Stolarsky results, see Theorem 2.1. We then generalize the evaluation of resultants of two two-term linear combinations of Chebyshev polynomials of the second kind to the sum of three term linear combinations of the same polynomials (see Theorem 2.2 and (2.11)). Section 3 contains similar results for Chebyshev polynomials of the first kind.

\section{Chebyshev polynomials of the second kind}

The Chebyshev polynomials of the second kind $\left\{U_{n}(x)\right\}$ are defined as

$$
U_{n}(x)=\frac{\sin (n+1) \theta}{\sin \theta},
$$

where $x=\cos \theta$ (see [13] and [1]). They solve the three term recurrence relation

$$
2 x y_{n}(x)=y_{n+1}(x)+y_{n-1}(x)
$$

under the initial conditions $U_{0}(x)=1, U_{1}(x)=2 x$. These polynomials satisfy the orthogonality relation

$$
\int_{-1}^{1} U_{n}(x) U_{m}(x) \sqrt{1-x^{2}} d x=\frac{\pi}{2} \delta_{m, n}
$$

Theorem 2.1 ( [6]). For $n \geq 2$, the resultant formula

$$
\operatorname{Res}\left\{U_{n}(x)+k U_{n-1}(x), U_{n-1}(x)+h U_{n-2}(x)\right\}=(-1)^{\frac{n(n-1)}{2}} 2^{n(n-1)} d_{n}(h, k)
$$

holds, with $d_{n}(x, y)=x^{n}\left[U_{n}\left(\frac{1+x y}{2 x}\right)-y U_{n-1}\left(\frac{1+x y}{2 x}\right)\right]$.

Proof. Let $r_{n}(x):=U_{n}(x)+k U_{n-1}(x)$ and $s_{n}(x):=U_{n}(x)+h U_{n-1}(x)$. Thus we need to evaluate $\operatorname{Res}\left\{r_{n}(x), s_{n-1}(x)\right\}$. It is not hard to see that

$$
s_{n-1}(x)=A_{n}(x) r_{n-1}(x)+B_{n}(x) r_{n}(x)
$$

holds with

$$
A_{n}(x)=\frac{h}{k} \frac{x+\frac{1+h k}{2 h}}{x+\frac{1+k^{2}}{2 k}}, \quad B_{n}(x)=\frac{k-h}{1+2 x k+k^{2}} .
$$

The polynomial $r_{n}(x)$ has degree $n$ with leading coefficient $2^{n}$. Thus $r_{n}(x)=$ $2^{n} \prod_{j=1}^{n}\left(x-x_{j}\right)$. Hence (2.2) shows that $s_{n-1}\left(x_{j}\right)=A_{n}\left(x_{j}\right) r_{n-1}\left(x_{j}\right)$, which implies

$$
\prod_{j=1}^{n} s_{n-1}\left(x_{j}\right)=\prod_{j=1}^{n} A_{n}\left(x_{j}\right) \prod_{j=1}^{n} r_{n-1}\left(x_{j}\right)=\Delta_{n} \frac{h^{n}}{k^{n}} \prod_{j=1}^{n} \frac{-x_{j}-\frac{1+h k}{2 h}}{-x_{j}-\frac{1+k^{2}}{2 k}}=\Delta_{n} \frac{h^{n}}{k^{n}} \frac{r_{n}\left(-\frac{1+h k}{2 h}\right)}{r_{n}\left(-\frac{1+k^{2}}{2 k}\right)},
$$


where $\Delta_{n}=\prod_{j=1}^{n} r_{n-1}\left(x_{j}\right)$. Now $U_{n}(-x)=(-1)^{n} U_{n}(x)$ and $U_{n}\left(\frac{1+k^{2}}{2 k}\right)=$ $\frac{k^{n+1}-k^{-n-1}}{k-k^{-1}}$ implies

$$
\prod_{j=1}^{n} s_{n-1}\left(x_{j}\right)=\Delta_{n} \frac{h^{n}}{k^{n}} \frac{U_{n}\left(\frac{1+h k}{2 h}\right)-k U_{n-1}\left(\frac{1+h k}{2 h}\right)}{U_{n}\left(\frac{1+k^{2}}{2 k}\right)-k U_{n-1}\left(\frac{1+k^{2}}{2 k}\right)} .
$$

This implies $\prod_{j=1}^{n} s_{n-1}\left(x_{j}\right)=\Delta_{n} d_{n}(h, k)$. Since $\left\{r_{n}(x)\right\}$ solves $(2.1)$, we apply Theorem 1.1 and conclude that $\Delta_{n}=(-1)^{\frac{n(n-1)}{2}} \prod_{j=1}^{n} 2^{n-2 j+1}=(-1)^{\frac{n(n-1)}{2}}$. This establishes Theorem 2.1.

Next we consider the more general linear combination of $\left\{U_{n}(x)\right\}$. Let

$$
\begin{aligned}
U_{n}(x ; a, k) & :=U_{n}(x)+(a x+k) U_{n-1}(x), \\
f(x) & :=1+(b x+h)(2 x+a x+k) \\
g(x) & :=1+(a x+k)(2 x+a x+k) .
\end{aligned}
$$

Theorem 2.2. We have

$\operatorname{Res}\left\{U_{n}(x ; a, k), U_{n-1}(x ; b, h)\right\}=\frac{(-1)^{\left(\begin{array}{c}n \\ 2\end{array}\right)}}{(2+a)^{2}} 2^{(n-1)(n-2)} \operatorname{Res}\left\{f(x), U_{n}(x ; a, k)\right\}$.

Proof. It can be readily verified that

$$
U_{n-1}(x ; b, h)=A_{n}(x) U_{n-1}(x ; a, k)+B_{n}(x) U_{n}(x ; a, k)
$$

holds with

$$
A_{n}(x)=\frac{1+(b x+h)(2 x+a x+k)}{1+(a x+k)(2 x+a x+k)}, \quad B_{n}(x)=\frac{(a-b) x+k-h}{1+(a x+k)(2 x+a x+k)} .
$$

Let $c_{1}, c_{2}$ and $d_{1}, d_{2}$ be the zeros of $f$ and $g$, respectively. Thus

$$
A_{n}(x)=\frac{b}{a} \frac{\left(x-c_{1}\right)\left(x-c_{2}\right)}{\left(x-d_{1}\right)\left(x-d_{2}\right)}
$$

From (2.4) we see that

$$
U_{n}(x ; a, k)=2^{n-1}(2+a) \prod_{j=1}^{n}\left(x-x_{j, n}\right) .
$$

Applying (2.8) in (2.6) and using (2.7) we arrive at

$$
\begin{aligned}
\prod_{j=1}^{n} U_{n-1}\left(x_{j, n} ; b, h\right) & =\prod_{j=1}^{n} A_{n}\left(x_{j, n}\right) \prod_{j=1}^{n} U_{n-1}\left(x_{j, n} ; a, k\right) \\
& =\Delta_{n} \frac{b^{n}}{a^{n}} \frac{\prod_{j=1}^{n}\left(c_{1}-x_{j, n}\right)\left(c_{2}-x_{j, n}\right)}{\prod_{j=1}^{n}\left(d_{1}-x_{j, n}\right)\left(d_{2}-x_{j, n}\right)}
\end{aligned}
$$


where $\Delta_{n}=\prod_{j=1}^{n} U_{n-1}\left(x_{j, n} ; a, k\right)$. This implies,

$$
\prod_{j=1}^{n} U_{n-1}\left(x_{j, n} ; b, h\right)=\Delta_{n} \frac{b^{n}}{a^{n}} \frac{U_{n}\left(c_{1} ; a, k\right) U_{n}\left(c_{2} ; a, k\right)}{U_{n}\left(d_{1} ; a, k\right) U_{n}\left(d_{2} ; a, k\right)} .
$$

We now compute $\Delta_{n}$. It is clear that $U_{0}(x ; a, k)=1$ and $U_{1}(x ; a, k)=(2+$ $a) x+k$. Moreover $U_{n}(x ; a, k)$ satisfies the three term recurrence relation (2.1). Applying Theorem 1.1 we conclude that $\Delta_{n}=(-1)^{\frac{n(n-1)}{2}} 2^{1-n}(2+a)^{n-1}$. From the above result and (2.9) we have the following equality:

$$
\prod_{j=1}^{n} U_{n-1}\left(x_{j, n} ; b, h\right)=(-1)^{\frac{n(n-1)}{2}}\left(1+\frac{a}{2}\right)^{n-1} \frac{b^{n}}{a^{n}} \frac{U_{n}\left(c_{1} ; a, k\right) U_{n}\left(c_{2} ; a, k\right)}{U_{n}\left(d_{1} ; a, k\right) U_{n}\left(d_{2} ; a, k\right)} .
$$

Clearly $(b(a+2))^{n} U_{n}\left(d_{1} ; a, k\right) U_{n}\left(d_{2} ; a, k\right)=\operatorname{Res}\left(f(x), U_{n}(x ; a, k)\right)$, and we only need to evaluate $U_{n}\left(d_{1} ; a, k\right) U_{n}\left(d_{2} ; a, k\right)$. Multiply (2.6) by $g$ and let $d$ be either $d_{1}$ or $d_{2}$. Thus $f(d) U_{n-1}(d ; a, k)+[(a-b) d+k-h] U_{n}(d ; a, k)=0$, which implies $U_{n}(d ; a, k)=(2 d+a d+k) U_{n-1}(d ; a, k)$, from which we conclude that $U_{n}(d ; a, k)=((a+2) d+k)^{n}$. Therefore $U_{n}\left(d_{1} ; a, k\right) U_{n}\left(d_{2} ; a, k\right)=\left(\frac{a+2}{a}\right)^{n}$. The result now follows from $(2.10)$.

Observe that

$$
\begin{aligned}
& U_{n}(x ; a, k)=\left(1+\frac{a}{2}\right) U_{n}(x)+k U_{n-1}+\frac{a}{2} U_{n-2}(x) \\
& U_{n}(x ; b, h)=\left(1+\frac{b}{2}\right) U_{n}(x)+h U_{n-1}+\frac{b}{2} U_{n-2}(x),
\end{aligned}
$$

hence one can evaluate in closed form the resultant of polynomials of the form $\sum_{j=0}^{2} c_{j} U_{n-j}$ and $\sum_{j=0}^{2} d_{j} U_{n-j}$.

Remark 2.1. Let $v_{n}(x)=\sum_{j=0}^{m} c_{j} U_{n-j}(x)$ and $w_{n}(x)=\sum_{j=0}^{m} d_{j} U_{n-j}(x)$. Then, in general, there exist polynomials $f, g$ and $h$ of degrees $m, m$ and $m-1$, respectively, such that

$$
f(x) w_{n-1}(x)=g(x) v_{n-1}(x)+h(x) v_{n}(x) .
$$

This is intuitively clear for the following reason. The left-hand side of (2.11) is a polynomial of degree $m+n-1$ and by repeatedly using (2.1) it can be expressed as $\sum_{k=0}^{3 m} \alpha_{k} U_{n+m-1-k}$ and there is no loss of generality in assuming $\alpha_{0}=1$, that is $f(x)=2^{m} x^{m}+\cdots$. By equating coefficients of various Chebyshev polynomials $U_{n}$ we find $3 m+1$ linear equations in the coefficients of $f, g$ and $h$. The total number of coefficents in $f, g$ and $h$ is $2(m+1)+m$ coefficients. Since one coefficient has already been specified we only have $3 m+1$ unknowns and $3 m+1$ equations, so the problem is doable in general. The case of the Chebyshev polynomials of the first kind is more transparent, see $\S 3$. 
Let $x_{1}, x_{2}, \ldots, x_{n}$ be the zeros of $v_{n}(x)$, that is

$$
v_{n}(x)=2^{n} \prod_{j=1}^{n}\left(x-x_{j}\right) .
$$

Moreover we let $f(x)=2^{m} \prod_{k=1}^{m}\left(x-f_{k}\right)$ and $g(x)=\gamma \prod_{k=1}^{m}\left(x-g_{k}\right)$. The fact

$$
\prod_{j=1}^{n} \frac{g\left(x_{j}\right)}{f\left(x_{j}\right)}=\frac{\gamma^{n}}{2^{m n}} \prod_{j=1}^{n} \prod_{k=1}^{m} \frac{\left(x_{j}-g_{k}\right)}{\left(x_{j}-f_{k}\right)}=\frac{\gamma^{n}}{2^{m n}} \prod_{k=1}^{m} \frac{v_{n}\left(g_{k}\right)}{v_{n}\left(f_{k}\right)},
$$

and (2.12) imply

$$
\prod_{j=1}^{n} w_{n-1}\left(x_{j}\right)=\prod_{j=1}^{n} \frac{g\left(x_{j}\right) v_{n-1}\left(x_{j}\right)}{f\left(x_{j}\right)}=\frac{\gamma^{n}}{2^{m n}} \prod_{k=1}^{m} \frac{v_{n}\left(g_{k}\right)}{v_{n}\left(f_{k}\right)} \Delta_{n},
$$

and $\Delta_{n}:=\prod_{j=1}^{n} v_{n-1}\left(x_{j}\right)$, which can be found from Theorem 1.1.

\section{Chebyshev polynomials of the first kind}

Chebyshev polynomials of the first kind are defined as

$$
T_{n}(x)=\cos n \theta, \quad x=\cos \theta,
$$

for $n=0,1,2, \ldots$ (see [13] and [1]). Indeed $T_{0}(x)=1, T_{1}(x)=x$ and the polynomials $\left\{T_{n}(x)\right\}$ satisfy the recurrence in (2.1). The first main result of this section is the following.

Theorem 3.1. Let $\left\{T_{n}(x)\right\}$ be the sequence of Chebyshev polynomials of first kind. Then,

$$
\begin{aligned}
& \operatorname{Res}\left\{T_{n}(x)+k T_{n-1}(x), T_{n-1}(x)+h T_{n-2}(x)\right\} \\
& =\frac{2^{n^{2}-3 n+3}}{(-1)^{\frac{n(n-1)}{2}}} h^{n}\left[T_{n}\left(\frac{1+h k}{2 h}\right)-k T_{n-1}\left(\frac{1+h k}{2 h}\right)\right] .
\end{aligned}
$$

Proof. Set

$$
r_{n}(x)=T_{n}(x)+k T_{n-1}(x), \quad s_{n}(x)=T_{n}(x)+h T_{n-1}(x) .
$$

Since $\left\{T_{n}(x)\right\}$ and $\left\{U_{n}(x)\right\}$ satisfy the same recurrence relation it follows that $s_{n-1}(x)=A_{n}(x) r_{n-1}(x)+B_{n}(x) r_{n}(x)$, where $A_{n}(x)$ and $B_{n}(x)$ are given by (2.3). From (3.1) we see that $r_{n}(x)$ is a polynomial of degree $n$ with leading coefficient 
$2^{n-1}$. Let $\left\{y_{j}\right\}_{j=1}^{n}$ be zeros of $r_{n}(x)$. Therefore $r_{n}(x)=2^{n-1} \prod_{j=1}^{n}\left(x-y_{j}\right)$, and $s_{n-1}\left(y_{j}\right)=A_{n}\left(y_{j}\right) r_{n-1}\left(y_{j}\right)$. This implies

$$
\begin{aligned}
\prod_{j=1}^{n} s_{n-1}\left(y_{j}\right) & =\frac{h^{n}}{k^{n}} \prod_{j=1}^{n} \frac{\left(-y_{j}-\frac{1+h k}{2 h}\right)}{\left(-y_{j}-\frac{1+k^{2}}{2 k}\right)} \prod_{j=1}^{n} r_{n-1}\left(y_{j}\right) \\
& =\Delta_{n} \frac{h^{n}}{k^{n}} \frac{r_{n}\left(-\frac{1+h k}{2 h}\right)}{r_{n}\left(-\frac{1+k^{2}}{2 k}\right)} \\
& =\Delta_{n} \frac{h^{n}}{k^{n}} \frac{T_{n}\left(-\frac{1+h k}{2 h}\right)+k T_{n-1}\left(-\frac{1+h k}{2 h}\right)}{T_{n}\left(-\frac{1+k^{2}}{2 k}\right)+k T_{n-1}\left(-\frac{1+k^{2}}{2 k}\right)},
\end{aligned}
$$

where $\Delta_{n}=\prod_{j=1}^{n} r_{n-1}\left(y_{j}\right)$. Recall that $T_{n}(-x)=(-1)^{n} T_{n}(x)$ and $T_{n}\left(\frac{z+z^{-1}}{2}\right)=$ $\frac{z^{n}+z^{-n}}{2}$. This leads to

$$
\prod_{j=1}^{n} s_{n-1}\left(y_{j}\right)=\frac{2 \Delta_{n} h^{n}}{1-k^{2}}\left[T_{n}\left(\frac{1+h k}{2 h}\right)-k T_{n-1}\left(\frac{1+h k}{2 h}\right)\right] .
$$

One can easily verify that the polynomials $\left\{r_{n}(x)\right\}$ satisfy the recurrence relation (2.1). Applying (1.3) and observing that $\Delta_{1}=1-k^{2}$ it follows that $\Delta_{n}=$ $(-1)^{\frac{n(n-1)}{2}} 2^{1-n}\left(1-k^{2}\right)$. Using this in (3.2) we have,

$$
\prod_{j=1}^{n} s_{n-1}\left(y_{j}\right)=\frac{(-1)^{\frac{n(n-1)}{2}} 2^{2-n} h^{n}}{1-k^{2}}\left[T_{n}\left(\frac{1+h k}{2 h}\right)-k T_{n-1}\left(\frac{1+h k}{2 h}\right)\right] .
$$

The theorem now follows from (1.1) and (3.3).

Next we consider the resultant of the following combination of Chebyshev polynomials of first kind. We let

$$
T_{n}(x ; a, k):=T_{n}(x)+(a x+k) T_{n-1}(x) .
$$

A calculation leads to the following expression for $T_{n-1}(x ; b, h)$ :

$$
T_{n-1}(x ; b, h)=A_{n}(x) T_{n-1}(x ; a, k)+B_{n}(x) T_{n}(x ; b, k)
$$

with $A_{n}$ and $B_{n}$ given by

$$
A_{n}(x)=\frac{1+(b x+h)(2 x+a x+k)}{1+(a x+k)(2 x+a x+k)}, \quad B_{n}(x)=\frac{(a-b) x+k-h}{1+(a x+k)(2 x+a x+k)} .
$$

This implies

$$
A_{n}(x)=\frac{b}{a} \frac{\left(x-c_{1}\right)\left(x-c_{2}\right)}{\left(x-d_{1}\right)\left(x-d_{2}\right)},
$$


where, as before, $c_{i}$ and $d_{i}$ for $i=1,2$ are the zeros of $f$ and $g$ defined in (2.5).

From (3.4) we observe that $T_{n}(x ; a, k)$ is a polynomial of degree $n$ with leading coefficient $2^{n-2}(2+a)$. Hence we can assume that $T_{n}(x ; a, k)=2^{n-2}(2+$ a) $\prod_{j=1}^{n}\left(x-y_{j, n}\right)$. The evaluation of $T_{n-1}(x ; b, h)$ at the zeros of $T_{n}(x ; a, k)$ is given by $T_{n-1}\left(y_{j, n} ; b, h\right)=A_{n}\left(y_{j, n}\right) T_{n-1}\left(y_{j, n} ; a, k\right)$. This together with (3.5) implies that

$$
\prod_{j=1}^{n} T_{n-1}\left(y_{j, n} ; b, h\right)=\frac{b^{n}}{a^{n}} \frac{\prod_{j=1}^{n}\left(c_{1}-y_{j, n}\right)\left(c_{2}-y_{j, n}\right)}{\prod_{j=1}^{n}\left(d_{1}-y_{j, n}\right)\left(d_{2}-y_{j, n}\right)} \prod_{j=1}^{n} T_{n-1}\left(y_{j, n} ; a, k\right) .
$$

It follows that

$$
\prod_{j=1}^{n} T_{n-1}\left(y_{j, n} ; b, h\right)=\frac{b^{n}}{a^{n}} \Delta_{n} \frac{T_{n}\left(c_{1} ; a, k\right) T_{n}\left(c_{2} ; a, k\right)}{T_{n}\left(d_{1} ; a, k\right) T_{n}\left(d_{2} ; a, k\right)},
$$

where $\Delta_{n}=\prod_{j=1}^{n} T_{n-1}\left(y_{j, n} ; a, k\right)$. As in the proof of Theorem 2.2 we apply $T_{0}(x ; a, k)=1$ and $T_{1}(x ; a, k)=(a+1) x+k$ to show that

$$
\begin{aligned}
\Delta_{n} & =\prod_{j=1}^{n} T_{n-1}\left(y_{j, n} ; a, k\right) \\
& =\left(2^{n-3}(2+a)\right)^{n} \prod_{j=1}^{n}\left(y_{j, n}-y_{1, n-1}\right) \cdots\left(y_{j, n}-y_{n-1, n-1}\right) \\
& =2^{-2}(2+a) \prod_{j=1}^{n-1} T_{n}\left(y_{j, n-1} ; a, k\right) \\
& =(-1)^{n-1}(2+a) 2^{-2} \Delta_{n-1} .
\end{aligned}
$$

We used the three term recurrence relation given above in the last equality. This inductively implies that

$$
\Delta_{n}=(-1)^{\frac{n(n-1)}{2}}(2+a)^{n-1} 2^{2-2 n} .
$$

Theorem 3.2. Let $T_{n}(x ; a, k)$ be defined by (3.4). Then,

$$
\operatorname{Res}\left\{T_{n}(x ; a, k), T_{n-1}(x ; b, h)\right\}=(-1)^{\frac{n(n-1)}{2}} 2^{n^{2}-5 n+4} \frac{\operatorname{Res}\left\{f, T_{n}(x ; a, k)\right\}}{(a+1)^{2}-k^{2}} .
$$

Proof. It follows from (1.1), (3.6) and (3.7) that the left-hand side of (3.8) equals

$$
(-1)^{\frac{n(n-1)}{2}}(2+a)^{2 n-2} 2^{n^{2}-5 n+4} \frac{b^{n}}{a^{n}} \frac{T_{n}\left(c_{1} ; a, k\right) T_{n}\left(c_{2} ; a, k\right)}{T_{n}\left(d_{1} ; a, k\right) T_{n}\left(d_{2} ; a, k\right)} .
$$


A calculation gives $T_{n}\left(d_{j} ; a, k\right)=[(a+2) d+k] T_{n-1}\left(d_{j} ; a, k\right), j=1,2$, and

$$
\begin{aligned}
& \operatorname{Res}\left\{g(x), T_{1}(x ; a, k)\right\}=(a+1+k)(a+1-k) \\
& \operatorname{Res}\left\{g(x), T_{2}(x ; a, k)\right\}=(a+2)^{2}(a+1+k)(a+1-k) .
\end{aligned}
$$

Hence Res $\left\{g, T_{n}(. ; a, k)\right\}=a^{n}(a+2)^{n} T_{n}\left(d_{1} ; a, k\right) T_{n}\left(d_{2} ; a, k\right)=(a+2)^{2 n-2}(a+$ $1+k)(a+1-k)$. This completes the proof.

Recall that the Chebyshev polynomials are special Jacobi polynomials. Indeed

$$
T_{n}(x)=\frac{n !}{\left(\frac{1}{2}\right)_{n}} P_{n}^{\left(-\frac{1}{2},-\frac{1}{2}\right)}(x), \quad U_{n}(x)=\frac{(n+1) !}{\left(\frac{3}{2}\right)_{n}} P_{n}^{\left(\frac{1}{2}, \frac{1}{2}\right)}(x),
$$

where $(a)_{n}:=a(a+1) \cdots(a+n-1)$ is a shifted factorial. The expansion formula $[11,(2)$, p. 262]

$$
\frac{(1-x)^{n}}{2^{n}(1+\alpha)_{n}}=\sum_{k=0}^{n} \frac{(-n)_{k}(1+\alpha+\beta+2 k)(1+\alpha+\beta)_{k}}{(\alpha+1)_{k}(1+\alpha+\beta)_{n+k+1}} P_{n}^{(\alpha, \beta)}(x)
$$

contains the expansions of powers of $1 \pm x$ in Chebyshev polynomials of the first and second kinds, since $P_{n}^{(\alpha, \beta)}(-x)=(-1)^{n} P_{n}^{(\beta, \alpha)}(x)$. The term $k=0$ in (3.9) when $\alpha=\beta=-\frac{1}{2}$ seems to be indeterminate, but it can be found by a limiting procedure to be $\frac{1}{n !}$. Thus

$$
\frac{(1-x)^{n}}{2^{n}\left(\frac{1}{2}\right)_{n}}=\frac{1}{n !}+2 \sum_{k=1}^{n} \frac{(-n)_{k} k !}{\left(\frac{1}{2}\right)_{k}(n+k) !} T_{n}(x) .
$$

We now discuss the case when

$$
\tilde{v}_{n}(x)=\sum_{j=0}^{m} c_{j} T_{n-j}(x), \quad \tilde{w}_{n}(x)=\sum_{j=0}^{m} d_{j} T_{n-j}(x), \quad c_{0}=d_{0}=1 .
$$

As per Remark 2.1, in general, there exist polynomials $f, g$ and $h$ of degrees $m$, $m$ and $m-1$, respectively, such that

$$
f(x) \tilde{w}_{n-1}(x)=g(x) \tilde{v}_{n-1}(x)+h(x) \tilde{v}_{n}(x) .
$$

In this case the analysis is made simpler by expanding $f, g$ and $h$ in powers of $1-x$, applying (3.10) and using $T_{n}(x) T_{m}(x)=\frac{1}{2}\left[T_{m+n}(x)+T_{m-n}(x)\right]$, to set up the linear system of equations satisfied by the coefficients of $f, g$ and $h$. The difference between this case and the case of Chebyshev polynomials of the second kind is that the linearization of products is more complicated.

Since $T_{n}(x)=2^{n-1} x^{n}+\cdots$, we let

$$
\tilde{v}_{n}(x)=2^{n-1} \prod_{j=1}^{n}\left(x-y_{j}\right), \quad f(x)=2^{m} \prod_{k=1}^{m}\left(x-f_{k}\right), \quad g(x)=\gamma \prod_{k=1}^{m}\left(x-g_{k}\right) .
$$


Indeed, $\prod_{j=1}^{n} \frac{g\left(x_{j}\right)}{f\left(x_{j}\right)}=\frac{\gamma^{n}}{2^{m n}} \prod_{k=1}^{m} \frac{\tilde{v}_{n}\left(g_{k}\right)}{\tilde{v}_{n}\left(f_{k}\right)}$ and (2.1) imply

$$
\prod_{j=1}^{n} \tilde{w}_{n-1}\left(x_{j}\right)=\frac{\gamma^{n}}{2^{m n}} \prod_{k=1}^{m} \frac{\tilde{v}_{n}\left(g_{k}\right)}{\tilde{v}_{n}\left(f_{k}\right)} \tilde{\Delta}_{n}, \quad \tilde{\Delta}_{n}:=\prod_{j=1}^{n} \tilde{v}_{n-1}\left(x_{j}\right) .
$$

Now $\tilde{\Delta}_{n}$ can be computed using Theorem 1.1 .

Acknowledgement. The authors are indebted to an anonymous referee for many suggestions about content and notation and for correcting misprints. These comments improved the presentation of our results.

\section{References}

[1] Andrews, G. E., Askey, R. A. and Roy, R., Special Functions. Cambridge: Cambridge Univ. Press 1999.

[2] Apostol, T. M., The resultants of the cyclotomic polynomials $F_{m}(a x)$ and $F_{n}(b x)$. Math. Comp. 29 (1975), $1-6$.

[3] Barnett, S., Polynomials and Linear Control Systems. New York: Marcel Dekker 1983.

[4] Barnett, S., Matrices in Control Theory (2nd ed.). Melbourne (FL): Krieger 1984.

[5] Dickson, L. E., New Course on the Theory of Equations. New York: Wiley 1939.

[6] Dilcher, K. and Stolarsky, K. B., Resultants and discriminants of Chebyshev and related polynomials. Trans. Amer. Math. Soc. 357 (2004), 965 - 981.

[7] Gelfand, I. M., Kapranov, M. M. and Zelevinsky, A. V., Discriminants, Resultants, and Multidimensional Determinants. Boston: Birkhäuser 1994.

[8] Gerber, H. U., Wronski's formula and the resultant of two polynomials. Amer. Math. Montly 91 (1984), $644-646$.

[9] Ismail, M. E. H., Discriminants and functions of the second kind of orthogonal polynomials. Results Math. 34 (1998), 132 - 149.

[10] Ismail, M. E. H., An electrostatic model for zeros of general orthogonal polynomials. Pacific J. Math. 193 (2000), 355 - 369.

[11] Rainville, E. D., Special Functions. New York: Macmillan 1960.

[12] Roberts, D. P., Discriminants of some Painlevé polynomials. In: Number Theory for the Millennium III (Urbana, IL, 2000). Natick (MA): A K Peters 2002, pp. $205-221$.

[13] Szegö, G., Orthogonal Polynomials (fourth ed.). Providence (RI): Amer. Math. Soc. 1975.

Received September 26, 2005; revised March 31, 2006 